\title{
Positive solutions for integral boundary value problem of two-term fractional differential equations
}

Mengrui $\mathrm{Xu}^{1}$ and Zhenlai Han ${ }^{1 *}$

*Correspondence:

hanzhenlai@163.com

'school of Mathematical Sciences,

University of Jinan, Jinan, P.R. China

\begin{abstract}
In this paper, we investigate a class of nonlinear two-term fractional differential equations involving two fractional orders $\delta \in(1,2]$ and $\tau \in(0, \delta)$ with integral boundary value conditions. By the Schauder fixed point theorem we obtain the existence of positive solutions based on the method of upper and lower solutions. Then we obtain the uniqueness result by the Banach contraction mapping principle. Examples are given to illustrate our main results.
\end{abstract}

MSC: 34A08; 34B18; 34A12

Keywords: Fractional differential equations; Boundary value problem; Positive solutions

\section{Introduction}

Fractional differential equations are an important tool to describe many processes and phenomena of science and engineering [1-3]. The boundary value problems of fractional differential equations have received widespread attention in recent years, and there are some attractive results obtained: see [4-9]. The theory of lower and upper solutions is known to be an effective method for proving the existence of solutions to fractional differential equations (see, e.g., [10-14]).

In this paper, we study positive solutions for the integral boundary value problems

$$
\begin{aligned}
& D^{\delta} x(t)+f(t, x(t))=D^{\tau} g(t, x(t)), \quad t \in(0,1), \\
& x(0)=0, \quad x(1)=\frac{1}{\Gamma(\delta-\tau)} \int_{0}^{1}(1-s)^{\delta-\tau-1} g(s, x(s)) d s,
\end{aligned}
$$

where $D^{\delta}$ and $D^{\tau}$ are the standard Riemann-Liouville derivatives, $1<\delta \leq 2,0<\tau<\delta$, $f, g:[0,1] \times[0,1] \rightarrow[0,+\infty)$ are given continuous functions, $g(t, x)$ is nondecreasing on $x$ for any $t \in[0,1]$, and $f$ is not required any monotone assumption.

In the literature, single-term fractional differential equations of the form $D^{\delta} x(t)=$ $f(t, x(t))$ have been studied by many researchers (see [15-23]). In practical problems the equation contains more than one fractional differential term, for example, the classical

(c) The Author(s) 2018. This article is distributed under the terms of the Creative Commons Attribution 4.0 International License (http://creativecommons.org/licenses/by/4.0/), which permits unrestricted use, distribution, and reproduction in any medium, provided you give appropriate credit to the original author(s) and the source, provide a link to the Creative Commons license, and indicate if changes were made. 
Bagley-Torvik equation [24]

$$
A D^{2} y(x)+B D^{\frac{3}{2}} y(x)+C y(x)=f(x)
$$

where $A, B, C$ are constants, and $f$ is a given function. This equation was introduced in 1984 as the mathematical model for the motion of thin plate in Newtonian fluid. The Langevin equation with the fractional derivatives of the form

$$
D^{\beta}\left(D^{\alpha}+\mu\right) x(t)=f(t)
$$

is another great example [25-27]. More generally, we can refer to $[6,10]$ on the equation of type $D^{\beta} x(t)=f\left(t, x(t), D^{\alpha} x(t)\right)$.

Recently, in the aspect of theories, there are some excellent results on the existence of solutions to two-term fractional differential equations. For example, Ibrahim et al. [28] studied the boundary value problem

$$
\left\{\begin{array}{l}
{ }^{c} D^{\alpha} u(t)-a^{c} D^{\beta} u(t)+f(t, u(t))=0, \quad 0<t<1, \\
u(0)=u_{0}, \quad u(1)=u_{1},
\end{array}\right.
$$

where ${ }^{c} D^{\alpha}$ and ${ }^{c} D^{\beta}$ are the Caputo fractional derivatives with $1<\alpha \leq 2$ and $1 \leq \beta<\alpha$. The existence and uniqueness of solutions were obtained by the Banach contraction principle and Krasnoselskii's fixed point theorem.

Staněk [29], applying the Schauder fixed point theorem, considered the existence, multiplicity, and uniqueness of solutions to the periodic boundary value problem

$$
\left\{\begin{array}{l}
{ }^{c} D^{\alpha} u(t)+q(t, u(t))^{c} D^{\beta} u(t)=f(t, u(t)), \quad 0<t<1, \\
u(0)=u(T) .
\end{array}\right.
$$

Agarwal et al. [6] investigated the singular fractional Dirichlet boundary value problem

$$
\left\{\begin{array}{l}
D^{\alpha} u(t)+f\left(t, u(t), D^{\mu} u(t)\right)=0 \\
u(0)=u(1)=0
\end{array}\right.
$$

By means of a fixed point theorem on cone, the existence of positive solutions was proved.

In recent paper [30], positivity results of the initial value problems

$$
\left\{\begin{array}{l}
{ }^{c} D^{\alpha} x(t)=f(t, x(t))+{ }^{c} D^{\alpha-1} g(t, x(t)), \quad 0<t \leq T, \\
x(0)=\theta_{1}>0, \quad x^{\prime}(0)=\theta_{2}>0,
\end{array}\right.
$$

were considered by using the method of upper and lower solutions, Schauder fixed point theorem, and Banach fixed point theorem. It is interesting that the upper and lower control functions $f$ need no any monotone requirement.

To the best of our knowledge, no paper has considered the existence of positive solutions for nonlinear fractional differential equations with integral boundary conditions 
(1.1)-(1.2). This equation has two nonlinear terms, one containing the fractional derivative. Compared to many two-term fractional differential equations, the form of the equation we considered is more general in a way. In addition, the other nonlinear term requires no any monotonicity, which can respond better to objective laws.

In this paper, we prove the existence of positive solutions to the boundary value problem (1.1)-(1.2) by the Schauder fixed point theorem and the method of upper and lower solutions. Then, we give a uniqueness result by the Banach contraction mapping principle.

The paper is organized as follows. Section 2 contains some necessary concepts and results. In Sect. 3, our main results on the existence of positive solutions are proved. Section 4 is devoted to study the uniqueness of positive solutions.

\section{Preliminaries}

In this section, we present some definitions and lemmas, which are required for our theorems.

Definition 2.1 ([1]) The fractional integral of order $\alpha>0$ of a function $f:(0,+\infty) \rightarrow \mathbb{R}$ is given by

$$
I^{\alpha} f(t)=\frac{1}{\Gamma(\alpha)} \int_{0}^{t}(t-s)^{\alpha-1} f(s) d s
$$

where $\Gamma(\alpha)$ is the gamma function, provided that the right-hand side is pointwise defined on $(0,+\infty)$.

Definition 2.2 ([1]) The Riemann-Liouville fractional derivative of order $\alpha>0$ of a continuous function $f:(0,+\infty) \rightarrow \mathbb{R}$ is given by

$$
D^{\alpha} f(t)=\frac{1}{\Gamma(n-\alpha)}\left(\frac{d}{d t}\right)^{n} \int_{0}^{t}(t-s)^{n-\alpha+1} f(s) d s,
$$

where $n=[\alpha]+1([\alpha]$ denotes the integer part of a number $\alpha)$, provided that the right-hand side is pointwise defined on $(0,+\infty)$.

Lemma $2.1([2])$ Let $\alpha>0$. If $\in L^{1}\left([a, b], \mathbb{R}^{N}\right)$ and $I^{n-\alpha} f \in A C^{n}\left([a, b], \mathbb{R}^{N}\right)$, then

$$
I^{\alpha}\left(D^{\alpha} f(t)\right)=f(t)-\sum_{j=1}^{n} \frac{f_{n-\alpha}^{(n-j)}(a)}{\Gamma(\alpha-j+1)} t^{\alpha-j}
$$

almost everywhere on $[a, b]$, where $n$ is the smallest integer greater than or equal to $\alpha$.

Lemma 2.2 ([3]) If $\alpha>0$ and $\beta>0$, then the equation

$$
I^{\alpha}\left(I^{\beta} f(t)\right)=I^{\alpha+\beta} f(t)
$$

is satisfied at almost every point $t \in[a, b]$ for $f \in L^{p}\left([a, b], \mathbb{R}^{N}\right)(1 \leq p \leq \infty)$. If $\alpha+\beta>1$, then relation (2.1) holds at any point of $[a, b]$. 
Lemma 2.3 ([3]) If $\alpha>0$ and $\beta>0$, then

$$
I^{\alpha} t^{\beta-1}=\frac{\Gamma(\beta)}{\Gamma(\beta+\alpha)} t^{\beta+\alpha-1} .
$$

Lemma 2.4 ([3]) Let $X$ be a Banach space, and let $\Omega \subset X$ be a convex closed bounded set. If $T: \Omega \rightarrow \Omega$ is a continuous operator such that $T \Omega \subset X$ and $T \Omega$ is relatively compact, then $T$ has at least one fixed point in $\Omega$.

Lemma 2.5 ([3]) Let $(X, d)$ be a complete metric space, and let $T: X \rightarrow X$ be a contraction mapping:

$$
d(T x, T y) \leq k d(x, y)
$$

where $0<k<1$, for all $x, y \in X$. Then there exists a unique fixed point $x$ of $T$ in $X: T x=x$.

Lemma 2.6 Let $x \in L^{1}([0,1], \mathbb{R})$ and $I^{2-\delta} x \in A C^{2}([0,1], \mathbb{R})$ with $1<\delta \leq 2$. Then $x$ is a solution of the boundary value problem (1.1)-(1.2) if and only if

$$
x(t)=\int_{0}^{1} H(t, s) f(s, x(s)) d s+\frac{1}{\Gamma(\delta-\tau)} \int_{0}^{t}(t-s)^{\delta-\tau-1} g(s, x(s)) d s,
$$

where

$$
H(t, s)= \begin{cases}\frac{[t(1-s)]^{\delta-1}-(t-s)^{\delta-1}}{\Gamma(\delta)}, & 0 \leq s \leq t \leq 1, \\ \frac{[t(1-s)]^{\delta-1}}{\Gamma(\delta)}, & 0 \leq t \leq s \leq 1 .\end{cases}
$$

Proof The proof is divided into two cases.

Case 1. $\tau \leq 1$. From Lemma 2.1, applying $I^{\delta}$ on both sides of (1.1), it follows that

$$
\begin{aligned}
x(t)+c_{1} t^{\delta-1}+c_{2} t^{\delta-2}+I^{\delta} f(t, x(t)) & =I^{\delta-\tau}\left(I^{\tau} D^{\tau} g(t, x(t))\right) \\
& =I^{\delta-\tau}\left(g(t, x(t))+c_{3} t^{\tau-1}\right),
\end{aligned}
$$

that is,

$$
\begin{gathered}
x(t)+c_{1} t^{\delta-1}+c_{2} t^{\delta-2}+\frac{1}{\Gamma(\delta)} \int_{0}^{t}(t-s)^{\delta-1} f(s, x(s)) d s \\
=\frac{1}{\Gamma(\delta-\tau)} \int_{0}^{t}(t-s)^{\delta-\tau-1} g(s, x(s)) d s+c_{3} \frac{\Gamma(\tau)}{\Gamma(\delta)} t^{\delta-1} .
\end{gathered}
$$

By the boundary condition (1.2) we have $c_{2}=0$ and

$$
c_{1}=-\frac{1}{\Gamma(\delta)} \int_{0}^{1}(1-s)^{\delta-1} f(s, x(s)) d s+c_{3} \frac{\Gamma(\tau)}{\Gamma(\delta)} .
$$

Case 2. $\tau>$ 1. As in Case 1, we can obtain

$$
x(t)+c_{1} t^{\delta-1}+c_{2} t^{\delta-2}+I^{\delta} f(t, x(t))=I^{\delta-\tau}\left(g(t, x(t))+c_{3} t^{\tau-1}+c_{4} t^{\tau-2}\right)
$$


and

$$
\begin{aligned}
x(t) & +c_{1} t^{\delta-1}+c_{2} t^{\delta-2}+\frac{1}{\Gamma(\delta)} \int_{0}^{t}(t-s)^{\delta-1} f(s, x(s)) d s \\
= & \frac{1}{\Gamma(\delta-\tau)} \int_{0}^{t}(t-s)^{\delta-\tau-1} g(s, x(s)) d s+c_{3} \frac{\Gamma(\tau)}{\Gamma(\delta)} t^{\delta-1}+c_{4} \frac{\Gamma(\tau-1)}{\Gamma(\delta-1)} t^{\delta-2} .
\end{aligned}
$$

According to boundary condition (1.2), we get $c_{2}=c_{4} \frac{\Gamma(\tau-1)}{\Gamma(\delta-1)}$ and

$$
c_{1}=-\frac{1}{\Gamma(\delta)} \int_{0}^{1}(1-s)^{\delta-1} f(s, x(s)) d s+c_{3} \frac{\Gamma(\tau)}{\Gamma(\delta)} .
$$

Therefore

$$
\begin{aligned}
x(t)= & \frac{1}{\Gamma(\delta)} \int_{0}^{1} t^{\delta-1}(1-s)^{\delta-1} f(s, x(s)) d s-\frac{1}{\Gamma(\delta)} \int_{0}^{t}(t-s)^{\delta-1} f(s, x(s)) d s \\
& +\frac{1}{\Gamma(\delta-\tau)} \int_{0}^{t}(t-s)^{\delta-\tau-1} g(s, x(s)) d s \\
= & \int_{0}^{1} H(t, s) f(s, x(s)) d s+\frac{1}{\Gamma(\delta-\tau)} \int_{0}^{t}(t-s)^{\delta-\tau-1} g(s, x(s)) d s .
\end{aligned}
$$

This process is reversible.

Lemma 2.7 ([17]) The function $H(t, s)$ defined by (2.4) satisfies:

(1) $H(t, s)>0$ for $t, s \in(0,1)$;

(2) $\max _{0 \leq t \leq 1} H(t, s)=H(s, s), s \in(0,1)$.

Let the Banach space $X=C([0,1])$ be endowed with the norm $\|x\|=\max _{0 \leq t \leq 1}|x(t)|$. Define

$$
P=\{u \in X: u(t)>0, t \in(0,1], u(0)=0\} .
$$

The positive solution we consider in this paper is a function such that $u \in P$.

To use the fixed point theorem, according to Lemma 2.6, we define the operator $T$ as

$$
T x(t)=\int_{0}^{1} H(t, s) f(s, x(s)) d s+\frac{1}{\Gamma(\delta-\tau)} \int_{0}^{t}(t-s)^{\delta-\tau-1} g(s, x(s)) d s .
$$

Then we have the following lemma.

Lemma 2.8 The operator $T: X \rightarrow X$ is completely continuous.

Proof Obviously, operator $T$ is continuous because of the continuity of $f$ and $g$. For any $\eta>0$, take $B_{\eta}=\{x \in X,\|x\| \leq \eta\}$. Then for any $t \in[0,1], x \in B_{\eta}$, there exists a constant $M_{1}$ 
such that $f(t, x), g(t, x) \leq M_{1}$ and

$$
\begin{aligned}
T x(t)= & \int_{0}^{1} H(t, s) f(s, x(s)) d s \\
& +\frac{1}{\Gamma(\delta-\tau)} \int_{0}^{t}(t-s)^{\delta-\tau-1} g(s, x(s)) d s \\
\leq & \int_{0}^{1} \frac{1}{\Gamma(\delta)}[s(1-s)]^{\delta-1} M_{1} d s+\frac{M_{1}}{\Gamma(\delta-\tau)} \\
\leq & \frac{M_{1}}{\Gamma(\delta)}+\frac{M_{1}}{\Gamma(\delta-\tau)} .
\end{aligned}
$$

Thus $T\left(B_{\eta}\right)$ is uniformly bounded.

For all $x \in B_{\eta}, t_{1}, t_{2} \in[0,1], t_{1}<t_{2}$, we get

$$
\begin{aligned}
\left|(T x)\left(t_{2}\right)-(T x)\left(t_{1}\right)\right| & \mid \frac{1}{\Gamma(\delta)} \int_{0}^{1}\left[t_{2}(1-s)\right]^{\delta-1} f(s, x(s)) d s+\frac{1}{\Gamma(\delta)} \int_{0}^{t_{2}}\left(t_{2}-s\right)^{\delta-1} f(s, x(s)) d s \\
& +\frac{1}{\Gamma(\delta-\tau)} \int_{0}^{t_{2}}\left(t_{2}-s\right)^{\delta-\tau-1} g(s, x(s)) d s-\frac{1}{\Gamma(\delta)} \int_{0}^{1}\left[t_{1}(1-s)\right]^{\delta-1} f(s, x(s)) d s \\
& -\frac{1}{\Gamma(\delta)} \int_{0}^{t_{1}}\left(t_{1}-s\right)^{\delta-1} f(s, x(s)) d s-\frac{1}{\Gamma(\delta-\tau)} \int_{0}^{t_{1}}\left(t_{1}-s\right)^{\delta-\tau-1} g(s, x(s)) d s \mid \\
\leq & \mid \frac{t_{2}^{\delta-1}-t_{1}^{\delta-1}}{\Gamma(\delta)} \int_{0}^{1}(1-s)^{\delta-1} f(s, x(s)) d s \\
& +\frac{1}{\Gamma(\delta)} \int_{0}^{t_{2}}\left(\left(t_{2}-s\right)^{\delta-1}-\left(t_{1}-s\right)^{\delta-1}\right) f(s, x(s)) d s+\int_{t_{2}}^{t_{1}}\left(t_{1}-s\right)^{\delta-1} f(s, x(s)) d s \\
& +\int_{0}^{t_{2}}\left(t_{2}-s\right)^{\delta-\tau-1}-\left(t_{1}-s\right)^{\delta-\tau-1} g(s, x(s)) d s+\int_{t_{2}}^{t_{1}}\left(t_{1}-s\right)^{\delta-\tau-1} g(s, x(s)) d s \mid \\
\leq & \left|t_{2}^{\delta-1}-t_{1}^{\delta-1}+t_{2}^{\delta}-t_{1}^{\delta}\right| \frac{M_{1}}{\Gamma(\delta+1)}+\left|t_{2}^{\delta-\tau}-t_{1}^{\delta-\tau}\right| \frac{M_{1}}{\Gamma(\delta-\tau+1)} .
\end{aligned}
$$

As $t_{1} \rightarrow t_{2}$, the right-hand side of the inequality tends to zero, which means that $T\left(B_{\eta}\right)$ is equicontinuous. By the Arzelà-Ascoli theorem $T: X \rightarrow X$ is compact.

Let $a, b \in \mathbb{R}^{+}$with $b>a$. For any $x \in[a, b]$, we definite the upper control function by

$$
U(t, x)=\sup \{f(t, \lambda): a \leq \lambda \leq x\}
$$

and the lower control function by

$$
L(t, x)=\inf \{f(t, \lambda): x \leq \lambda \leq b\} .
$$

It is clear that $U(t, x)$ and $L(t, x)$ are nondecreasing on $x$ and

$$
L(t, x) \leq f(t, x) \leq U(t, x)
$$


Definition 2.3 Let $a, b \in \mathbb{R}^{+}$with $b>a$, and let $\bar{x}, \underline{x} \in P$ with $a \leq \underline{x}(t) \leq \bar{x}(t) \leq b$ satisfy

$$
\begin{aligned}
& \bar{x}(t) \geq \int_{0}^{1} H(t, s) U(s, \bar{x}(s)) d s+\frac{1}{\Gamma(\delta-\tau)} \int_{0}^{t}(t-s)^{\delta-\tau-1} g(s, \bar{x}(s)) d s, \\
& \underline{x}(t) \leq \int_{0}^{1} H(t, s) L(s, \underline{x}(s)) d s+\frac{1}{\Gamma(\delta-\tau)} \int_{0}^{t}(t-s)^{\delta-\tau-1} g(s, \underline{x}(s)) d s
\end{aligned}
$$

for all $t \in(0,1]$. Then the functions $\bar{x}$ and $\underline{x}$ are called a pair of upper and lower solutions of the boundary value problem (1.1)-(1.2).

\section{Existence of positive solutions}

In this section, we establish the existence of positive solutions for the boundary value problem (1.1)-(1.2) by the Schauder fixed point theorem based on the method of upper and lower solutions.

Theorem 3.1 Assume that $\bar{x}$ and $\underline{x}$ are a pair of upper and lower solutions of the boundary value problem (1.1)-(1.2). Then the boundary value problem (1.1)-(1.2) has at least one positive solution $x \in X$, and $\underline{x}(t) \leq x(t) \leq \bar{x}(t), t \in[0,1]$.

Proof Let

$$
K=\{x \in P: \underline{x}(t) \leq x(t) \leq \bar{x}(t), t \in[0,1]\} .
$$

Clearly, $\|x\| \leq b$. Thus $K \subset X$ is convex, bounded, and closed. By Lemma 2.8 the operator $T: X \rightarrow X$ is completely continuous. We only need to prove that $T(K) \subset K$. Let $x \in K$. Then, by the definitions of upper and lower solutions,

$$
\begin{aligned}
(T x)(t) & =\int_{0}^{1} H(t, s) f(s, x(s)) d s+\frac{1}{\Gamma(\delta-\tau)} \int_{0}^{t}(t-s)^{\delta-\tau-1} g(s, x(s)) d s \\
& \leq \int_{0}^{1} H(t, s) U(s, x(s)) d s+\frac{1}{\Gamma(\delta-\tau)} \int_{0}^{t}(t-s)^{\delta-\tau-1} g(s, x(s)) d s \\
& \leq \int_{0}^{1} H(t, s) U(s, \bar{x}(s)) d s+\frac{1}{\Gamma(\delta-\tau)} \int_{0}^{t}(t-s)^{\delta-\tau-1} g(s, \bar{x}(s)) d s \\
& \leq \bar{x}(t)
\end{aligned}
$$

and

$$
\begin{aligned}
(T x)(t) & =\int_{0}^{1} H(t, s) f(s, x(s)) d s+\frac{1}{\Gamma(\delta-\tau)} \int_{0}^{t}(t-s)^{\delta-\tau-1} g(s, x(s)) d s \\
& \geq \int_{0}^{1} H(t, s) L(s, x(s)) d s+\frac{1}{\Gamma(\delta-\tau)} \int_{0}^{t}(t-s)^{\delta-\tau-1} g(s, x(s)) d s \\
& \geq \int_{0}^{1} H(t, s) L(s, \underline{x}(s)) d s+\frac{1}{\Gamma(\delta-\tau)} \int_{0}^{t}(t-s)^{\delta-\tau-1} g(s, \underline{x}(s)) d s \\
& \geq \underline{x}(t) .
\end{aligned}
$$


Then $T: K \rightarrow K$ is a compact mapping. By the Schauder fixed point theorem, $T$ has at least one fixed point $x \in K$. Therefore, the boundary value problem (1.1)-(1.2) has at least one positive solution $x \in K$ with $\underline{x}(t) \leq x(t) \leq \bar{x}(t), t \in[0,1]$.

Corollary 3.1 Assume that there exist continuous functions $k_{1}, k_{2}, k_{3}$, and $k_{4}$ such that

$$
\begin{array}{ll}
0 \leq k_{1}(t) \leq f(t, u) \leq k_{2}(t)<\infty, & (t, u) \in[0,1] \times[0,+\infty), \\
0 \leq k_{3}(t) \leq g(t, u) \leq k_{4}(t)<\infty, & (t, u) \in[0,1] \times[0,+\infty),
\end{array}
$$

and one of $k_{1}(t)$ and $k_{3}(t)$ is not identically equal to 0 . Then the boundary value problem (1.1)-(1.2) has at least one positive solution $x \in P$, and

$$
\begin{aligned}
& x(t) \geq \int_{0}^{1} H(t, s) k_{1}(s) d s+\frac{1}{\Gamma(\delta-\tau)} \int_{0}^{t}(t-s)^{\delta-\tau-1} k_{3}(s) d s, \\
& x(t) \leq \int_{0}^{1} H(t, s) k_{2}(s) d s+\frac{1}{\Gamma(\delta-\tau)} \int_{0}^{t}(t-s)^{\delta-\tau-1} k_{4}(s) d s .
\end{aligned}
$$

Proof Consider the boundary value problem

$$
\left\{\begin{array}{l}
D^{\delta} y(t)+k_{2}(t)=D^{\tau} k_{4}(t), \\
y(0)=0, \quad y(1)=\frac{1}{\Gamma(\delta-\tau)} \int_{0}^{1}(1-s)^{\delta-\tau-1} k_{4}(s) d s .
\end{array}\right.
$$

Then (3.7) has a positive solution

$$
y(t)=\int_{0}^{1} H(t, s) k_{2}(s) d s+\frac{1}{\Gamma(\delta-\tau)} \int_{0}^{t}(t-s)^{\delta-\tau-1} k_{4}(s) d s .
$$

From the definition of a control function (2.12)-(2.13) we have

$$
k_{1}(t) \leq L(t, y) \leq U(t, y) \leq k_{2}(t), \quad(t, y) \in[0,1] \times[a, b],
$$

where $a, b$ are the minimum and maximum of $y(t)$ on $[0,1]$. Therefore this implies that

$$
y(t) \geq \int_{0}^{1} H(t, s) U(s, y(s)) d s+\frac{1}{\Gamma(\delta-\tau)} \int_{0}^{t}(t-s)^{\delta-\tau-1} g(s, y(s)) d s d s .
$$

Clearly, (3.8) is an upper solution of the boundary value problem (1.1)-(1.2). In the same way, we can conclude that

$$
z(t)=\int_{0}^{1} H(t, s) k_{1}(s) d s+\frac{1}{\Gamma(\delta-\tau)} \int_{0}^{t}(t-s)^{\delta-\tau-1} k_{3}(s) d s
$$

is a lower solution of the boundary value problem (1.1)-(1.2). By Theorem 3.1 the boundary value problem (1.1)-(1.2) has at least one positive solution $x \in P$ with $z(t) \leq x(t) \leq$ $y(t)$.

Corollary 3.2 Let (3.4) and $f(t, u) \geq k_{1}(t) \geq 0, t \in[0,1]$, hold with at least one of $k_{1}(t)$ and $k_{3}(t)$ not identically zero. Assume that $f(t, u)$ converges uniformly on $[0,1]$ to $k(t)$ as 
$u \rightarrow \infty$. Then the boundary value problem (1.1)-(1.2) has at least one positive solution on $P$.

Proof By assumption there exist $N, M_{2}>0$ such that

$$
|f(t, u)-k(t)|<N, \quad u>M_{2}, t \in[0,1]
$$

that is,

$$
0 \leq f(t, u) \leq k(t)+N, \quad u>M_{2}, t \in[0,1]
$$

Let $M_{3}=\max _{0 \leq t \leq 1,0 \leq u \leq M_{2}} f(t, u)$. Then, we have

$$
k_{1}(t) \leq f(t, u) \leq k(t)+N+M_{3}, \quad t \in[0,1], u \in[0,+\infty)
$$

Thus, by Corollary 3.1, the boundary value problem (1.1)-(1.2) has at least one positive solution $x \in P$ such that

$$
x(t) \geq \int_{0}^{1} H(t, s) k_{1}(s) d s+\frac{1}{\Gamma(\delta-\tau)} \int_{0}^{t}(t-s)^{\delta-\tau-1} k_{3}(s) d s
$$

and

$$
x(t) \leq \int_{0}^{1} H(t, s) k(s) d s+\frac{\left(N+M_{3}\right)\left(t^{\delta-1}-t^{\delta}\right)}{\Gamma(\delta+1)}+\frac{1}{\Gamma(\delta-\tau)} \int_{0}^{t}(t-s)^{\delta-\tau-1} k_{4}(s) d s .
$$

For simplicity, we denote

$$
M(t, \alpha, \beta)=\frac{\alpha\left(t^{\delta-1}-t^{\delta}\right)}{\Gamma(\delta+1)}+\frac{\beta t^{\delta-\tau}}{\Gamma(\delta-\tau+1)}
$$

\section{Corollary 3.3 Assume that}

$$
\begin{aligned}
& \lim _{u \rightarrow \infty} \max _{t \in[0,1]} \frac{f(t, u)}{u}<a_{1}, \\
& \lim _{u \rightarrow \infty} \max _{t \in[0,1]} \frac{g(t, u)}{u}<a_{2},
\end{aligned}
$$

where $a_{1}, a_{2} \in \mathbb{R}^{+}$with $\max _{t \in[0,1]} M\left(t, a_{1}, a_{2}\right)<1$, and $f(t, u) \geq c_{1}, g(t, u) \geq c_{2}, t \in[0,1], u \in$ $[0, \infty)$, where $c_{1}, c_{2} \geq 0$ with $c_{1}{ }^{2}+c_{2}{ }^{2} \neq 0$. Then the boundary value problem (1.1)-(1.2) has at least one positive solution $x \in P$.

Proof By (3.11) there exist constants $N_{1}, N_{2}, a_{1}, a_{2}>0$ such that

$$
f(t, u) \leq a_{1} u, \quad u>N_{1}, t \in[0,1]
$$

and

$$
g(t, u) \leq a_{2} u, \quad u>N_{2}, t \in[0,1]
$$


Let $b_{1}=\max _{t \in[0,1], u \in\left[0, N_{1}\right]} f(t, u)$ and $b_{2}=\max _{t \in[0,1], u \in\left[0, N_{2}\right]} g(t, u)$. Then we have

$$
f(t, u) \leq a_{1} u+b_{1}
$$

and

$$
g(t, u) \leq a_{2} u+b_{2}
$$

for $t \in[0,1]$ and $u \in[0, \infty)$.

Consider the boundary value problem

$$
\left\{\begin{array}{l}
D^{\delta} x(t)+a_{1} x(t)+b_{1}=D^{\tau}\left(a_{2} x(t)+b_{2}\right), \quad t \in(0,1), \\
x(0)=0, \quad x(1)=\frac{a_{2}}{\Gamma(\delta-\tau)} \int_{0}^{1}(1-s)^{\delta-\tau-1} x(s) d s+\frac{b_{2}}{\Gamma(\delta-\tau+1)} .
\end{array}\right.
$$

Then $x \in L^{1}([0,1], \mathbb{R})$ and $I^{2-\delta} x \in A C^{2}([0,1], \mathbb{R})$ is a solution of (3.13) if and only if

$$
\begin{aligned}
x(t) & =\int_{0}^{1} H(t, s)\left(a_{1} x(s)+b_{1}\right) d s+\frac{1}{\Gamma(\delta-\tau)} \int_{0}^{t}(t-s)^{\delta-\tau-1}\left(a_{2} x(s)+b_{2}\right) d s \\
& =a_{1} \int_{0}^{1} H(t, s) x(s) d s+\frac{a_{2}}{\Gamma(\delta-\tau)} \int_{0}^{t}(t-s)^{\delta-\tau-1} x(s) d s+M\left(t, b_{1}, b_{2}\right) .
\end{aligned}
$$

Define the operator $T_{1}: X \rightarrow X$ as

$$
\left(T_{1} x\right)(t)=a_{1} \int_{0}^{1} H(t, s) x(s) d s+\frac{a_{2}}{\Gamma(\delta-\tau)} \int_{0}^{t}(t-s)^{\delta-\tau-1} x(s) d s+M\left(t, b_{1}, b_{2}\right), \quad t \in[0,1] .
$$

By the proof of Lemma 2.8 operator $T_{1}$ is completely continuous in $X$. Let

$$
\gamma=\max _{t \in[0,1]}\left\{\frac{M\left(t, b_{1}, b_{2}\right)}{1-M\left(t, a_{1}, a_{2}\right)}\right\} .
$$

If $x \in B_{\gamma}=\{x \in X:\|x\| \leq \gamma\}$, then

$$
\begin{aligned}
\left|\left(T_{1} x\right)(t)\right| \leq & a_{1}\|x\| \int_{0}^{1} H(t, s) d s+b_{1} \int_{0}^{1} H(t, s) d s \\
& +\frac{a_{2}\|x\|}{\Gamma(\delta-\tau)} \int_{0}^{t}(t-s)^{\delta-\tau-1} d s+\frac{b_{2} t^{\delta-\tau}}{\Gamma(\delta-\tau+1)} \\
= & M\left(t, a_{1}, a_{2}\right)\|x\|+M\left(t, b_{1}, b_{2}\right) \\
\leq & \gamma .
\end{aligned}
$$

Thus $T_{1}: B_{\gamma} \rightarrow B_{\gamma}$ is a compact operator. Hence, by the Schauder fixed theorem, $T_{1}$ has at least one fixed point in $B_{\gamma}$, and (3.13) has at least one positive solution $\bar{x}(t)$ with

$$
\bar{x}(t)=a_{1} \int_{0}^{1} H(t, s) \bar{x}(s) d s+\frac{a_{2}}{\Gamma(\delta-\tau)} \int_{0}^{t}(t-s)^{\delta-\tau-1} \bar{x}(s) d s+M\left(t, b_{1}, b_{2}\right)
$$


By the definition of a control function we get

$$
\bar{x}(t) \geq \int_{0}^{1} H(t, s) U(s, \bar{x}(s)) d s+\frac{1}{\Gamma(\delta-\tau)} \int_{0}^{t}(t-s)^{\delta-\tau-1} g(s, \bar{x}(s)) d s .
$$

Then $\bar{x}$ is an upper positive solution of the boundary value problem (1.1)-(1.2). Similarly,

$$
\underline{x}(t)=M\left(t, c_{1}, c_{2}\right)
$$

is a lower solution of the boundary value problem (1.1)-(1.2). By Theorem 3.1 the boundary value problem (1.1)-(1.2) has at least one positive solution $x \in P$, and $\underline{x}(t) \leq x(t) \leq$ $\bar{x}(t)$.

Example 3.1 Consider the boundary value problem

$$
\left\{\begin{array}{l}
D^{\frac{5}{4}} x(t)+\frac{\Gamma(9 / 4) \sin x(t)}{2}+t+1=\frac{\Gamma(3 / 2)}{2} D^{\frac{3}{4}}(x(t)+\cos t+1), \quad t \in(0,1), \\
x(0)=0, \quad x(1)=\frac{1}{4} \int_{0}^{1}(1-s)^{-1 / 2}(x(s)+\cos s+1) d s
\end{array}\right.
$$

where $f(t, x)=\frac{\Gamma(9 / 4) \sin x}{2}+t+1$ and $g(t, x)=\frac{\Gamma(3 / 2)}{2}(x+\cos t+1)$. We can see that $g$ is nondecreasing in $x$ and that

$$
\begin{aligned}
& \lim _{x \rightarrow \infty} \max _{t \in[0,1]} \frac{f(t, x)}{x} \leq \frac{\Gamma(9 / 4)}{2}, \\
& \lim _{x \rightarrow \infty} \max _{t \in[0,1]} \frac{g(t, x)}{x} \leq \frac{\Gamma(3 / 2)}{2},
\end{aligned}
$$

and $\max _{t \in[0,1]} M\left(t, \frac{\Gamma(9 / 4)}{2}, \frac{\Gamma(3 / 2)}{2}\right)<1$. By Corollary 3.3, (3.18) has at least one positive solution.

\section{Uniqueness of positive solution}

In this section, we prove the uniqueness of a positive solution for the boundary value problem (1.1)-(1.2) by the Banach contraction mapping principle. In particular, the monotonicity of $g(t, x)$ is dispensable.

Theorem 4.1 Assume that $f+g \not \equiv 0$ and that there are constants $L_{1}, L_{2}>0$ such that

$$
\begin{aligned}
& |f(t, x)-f(t, y)| \leq L_{1}\|x-y\|, \\
& |g(t, x)-g(t, y)| \leq L_{2}\|x-y\|
\end{aligned}
$$

for all $t \in[0,1]$ and $x, y \in[0,+\infty)$. Then, if

$$
\max _{t \in[0,1]} M\left(t, L_{1}, L_{2}\right)<1
$$

then the boundary value problem (1.1)-(1.2) has a unique positive solution on P. 
Proof We will prove the $T$ defined by (2.10) is a contraction mapping on $P$. Clearly, if $x \in P$, then $T x \in P$. In fact, for any $x, y \in[0,+\infty)$, we get

$$
\begin{aligned}
|(T x)(t)-(T y)(t)| & \\
\leq & \int_{0}^{1} H(t, s)|f(s, x(s))-f(s, y(s))| d s \\
\quad & +\frac{1}{\Gamma(\delta-\tau)} \int_{0}^{t}(t-s)^{\delta-\tau-1}|g(s, x(s))-g(s, y(s))| d s \\
\leq & \int_{0}^{1} H(t, s) L_{1}\|x-y\| d s+\frac{1}{\Gamma(\delta-\tau)} \int_{0}^{t}(t-s)^{\delta-\tau-1} L_{2}\|x-y\| d s \\
= & M\left(t, L_{1}, L_{2}\right)\|x-y\| .
\end{aligned}
$$

Hence, $T$ is a contraction mapping on $P$. Therefore, by the Banach contraction mapping principle, $T$ has a unique fixed point on $P$, and the boundary value problem (1.1)-(1.2) has a unique positive solution on $P$.

Remark 4.1 The conditions of Corollary 3.3 imply the conditions of Theorem 4.1, and thus Corollary 3.3 also concludes the uniqueness of a positive solution.

Example 4.1 Consider the boundary value problem

$$
\left\{\begin{array}{l}
D^{\frac{5}{4}} x(t)+t^{2}+\frac{t x(t)}{3+x(t)}=D^{\frac{1}{8}}\left(1+t+\frac{x(t)}{2+x(t)}\right), \quad t \in(0,1), \\
x(0)=0, x(1)=\frac{1}{\Gamma(9 / 8)} \int_{0}^{1}(1-s)^{1 / 8}\left(1+s+\frac{x(s)}{2+x(s)}\right) d s,
\end{array}\right.
$$

where $f(t, x)=t^{2}+\frac{t x}{3+x}$ and $g(t, x)=1+t+\frac{x}{2+x}$. Then

$$
|f(t, x)-f(t, y)|=\left|\frac{t x}{3+x}-\frac{t y}{3+y}\right| \leq \frac{1}{3}|x-y|
$$

and

$$
|g(t, x)-g(t, y)|=\left|\frac{x}{2+x}-\frac{y}{2+y}\right| \leq \frac{1}{2}|x-y|
$$

for all $t \in[0,1]$ and $x, y \in[0,+\infty)$. Since $M\left(t, \frac{1}{3}, \frac{1}{2}\right)<1$, by Theorem $4.1,(4.2)$ has a unique positive solution.

Acknowledgements

The authors sincerely thank the reviewers for their valuable suggestions and useful comments that have led to the present improved version of the original manuscript.

\section{Funding}

This research is supported by the Natural Science Foundation of China (61703180) and by Shandong Provincial Natural Science Foundation (ZR2017MA043).

\section{Availability of data and materials}

The authors declare that all data and material in the paper are available and veritable. 


\section{Authors' contributions}

The authors declare that the study was realized in collaboration with the same responsibility. Both authors read and approved the final manuscript.

\section{Publisher's Note}

Springer Nature remains neutral with regard to jurisdictional claims in published maps and institutional affiliations.

Received: 18 March 2018 Accepted: 21 June 2018 Published online: 28 June 2018

\section{References}

1. Podlubny, I.: Fractional Differential Equations. Academic Press, New York (1999)

2. Kilbas, A., Srivastava, H., Trujillo, J.: Theory and Applications of Fractional Differential Equations. Elsevier, The Netherlands (2006)

3. Zhou, Y.: Basic Theory of Fractional Differential Equations. World Scientific, Singapore (2014)

4. Zhao, Y., Sun, S., Han, Z., Zhang, M.: Positive solutions for boundary value problems of nonlinear fractional differential equations. Appl. Math. Comput. 217, 6950-6958 (2011)

5. Wang, Y., Liu, L.: Positive solutions for a class of fractional 3-point boundary value problems at resonance. Adv. Differ. Equ. 2017, 7 (2017)

6. Agarwal, R.P., O'Regan, D., Staněk, S.: Positive solutions for Dirichlet problems of singular nonlinear fractiona differential equations. J. Math. Anal. Appl. 371, 57-68 (2010)

7. Zhang, L., Zheng, Z.: Lyapunov type inequalities for the Riemann-Liouville fractional differential equations of higher order. Adv. Differ. Equ. 2017, 270 (2017)

8. Cui, Y., Ma, W., Sun, Q., Su, X.: New uniqueness results for boundary value problem of fractional differential equation. Nonlinear Anal.: Model. Control 23, 31-39 (2018)

9. Cui, Y., Sun, Q., Su, X.: Monotone iterative technique for nonlinear boundary value problems of fractional order $p \in(2,3]$. Adv. Differ. Equ. 2017, 248 (2017)

10. Liu, X., Jia, M.: Multiple solutions for fractional differential equations with nonlinear boundary conditions. Comput. Math. Appl. 59, 2880-2886 (2010)

11. Liu, X., Jia, M., Ge, W.: The method of lower and upper solutions for mixed fractional four-point boundary value problem with p-Laplacian operator. Appl. Math. Lett. 65, 56-62 (2017)

12. Jia, M., Liu, X.: Multiplicity of solutions for integral boundary value problems of fractional differential equations with upper and lower solutions. Appl. Math. Comput. 232, 313-323 (2014)

13. Liu, X., Jia, M.: The method of lower and upper solutions for the general boundary value problems of fractional differential equations with p-Laplacian. Adv. Differ. Equ. 2018, 28 (2018)

14. Bai, Z., Zhang, S., Sun, S., Yin, C.: Monotone iterative method for fractional differential equations. Electron. J. Differ. Equ. 2016, 6 (2016)

15. Zhao, Y., Sun, S., Han, Z., Li, Q.: The existence of multiple positive solutions for boundary value problems of nonlinear fractional differential equations. Commun. Nonlinear Sci. Numer. Simul. 16, 2086-2097 (2011)

16. Yan, R., Sun, S., Sun, Y., Han, Z.: Boundary value problems for fractional differential equations with nonlocal boundary conditions. Adv. Differ. Equ. 2013, 176 (2013)

17. Bai, Z., Lü, H.: Positive solutions for boundary value problem of nonlinear fractional differential equation. J. Math. Anal. Appl. 311, 495-505 (2005)

18. Bai, Z:: On positive solutions of a nonlocal fractional boundary value problem. Nonlinear Anal., Theory Methods Appl. 72, 916-924 (2010)

19. Cabada, A., Hamdi, Z.: Nonlinear fractional differential equations with integral boundary value conditions. Appl. Math. Comput. 228, 251-257 (2012)

20. Zou, Y., He, G.: On the uniqueness of solutions for a class of fractional differential equations. Appl. Math. Lett. 74, 68-73 (2017)

21. Xu, X., Jiang, D., Yuan, C.: Multiple positive solutions for the boundary value problem of a nonlinear fractional differential equation. Nonlinear Anal. 71, 4676-4688 (2009)

22. Feng, W., Sun, S., Li, X., Xu, M.: Positive solutions to fractional boundary value problems with nonlinear boundary conditions. Bound. Value Probl. 2014, 225 (2014)

23. Hao, X., Wang, H., Liu, L., Cui, Y.: Positive solutions for a system of nonlinear fractional nonlocal boundary value problems with parameters and p-Laplacian operator. Bound. Value Probl. 2017, 182 (2017)

24. Torvik, P.J., Bagley, R.L.: On the appearance of the fractional derivative in the behavior of real materials. J. Appl. Mech. 51, 725-728 (1984)

25. Coffey, W.T., Kalmykov, Y.P., Waldron, J.T.: The Langevin Equation, 2nd edn. World Scientific, Singapore (2004)

26. Lutz, E.: Fractional Langevin equation. Phys. Rev. E 64, 051106 (2001)

27. Lim, S.C., Li, M., Teo, L.P.: Langevin equation with two fractional orders. Phys. Lett. A 372, 6309-6320 (2008)

28. Ibrahim, B.H.E., Dong, Q., Fan, Z.: Existence for boundary value problems of two-term Caputo fractional differential equations. J. Nonlinear Sci. Appl. 10, 511-520 (2017)

29. Staněk, S.: Periodic problem for two-term fractional differential equations. Fract. Calc. Appl. Anal. 20, 662-678 (2017)

30. Boulares, H., Ardjouni, A., Laskri, Y.: Positive solutions for nonlinear fractional differential equations. Positivity 21 $1201-1212(2017)$ 